\title{
SPM Characterization of Titanium Disilicide Nanostructures Grown on a Ni-Modified Si(100) Substrate
}

\author{
W. Koczorowski*, M. Bazarnik and R. Czajka \\ Institute of Physics, Poznań University of Technology, Nieszawska 13A, 60-965 Poznań, Poland
}

(Received December 13, 2010; in final form March 15, 2011)

\begin{abstract}
The paper presents our recent investigations of the early stage growth of titanium silicides on a modified $\mathrm{Si}(100)$ substrate. The substrate was modified by deposition of a minute amount of Ni atoms, and the subsequent flash annealing. This process led to the creation of parallel defect lines on the $\mathrm{Si}(100)$ surface. We expected $\mathrm{TiSi}_{2}$ to form elongated structures on top and/or between the defect lines. Though this idea failed, stable nanostructures were observed and characterized using scanning probe microscopy (scanning tunneling microscopy and atomic force microscopy) methods.
\end{abstract}

PACS: 68.37.Ef, 68.37.Ps

\section{Introduction}

The miniaturization process in electronics needs smaller and smaller functional nanostructures grown on well characterized substrates. The most popular in electronic industry, silicon substrates can undergo versatile reconstructions due to doping with metals $[1,2]$. These modified substrates can serve as a template for nanostructures growth, e.g. metal silicides. Therefore, the detailed characterization of the surface (at the atomic scale) and nanostructures at the very early stage of their growth is necessary to determine their local electronic properties. These properties used to differ from bulk material properties due to quantum size effects (QSE) and influence of local arrangement of substrate atoms, especially presence of defects $[3,4]$. Scanning probe microscopy and spectroscopy (SPM/S) seem to be the most suitable methods to investigate such systems, where the structure and electronic properties may differ at the nano- or even subnano-meter scale [5].

\section{Characterization of the investigated system}

The clean $\mathrm{Si}(100)$ substrate was observed and characterized by Hamers et al. at the end of the 1980s [6, 7]. The procedure to obtain a clean substrate requires complex chemical procedures of ex situ chemical cleaning followed by flash annealing under UHV conditions at a pressure below $2 \times 10^{-10}$ Tr to obtain finally the $2 \times 1$ reconstruction [8]. Pairs of silicon atoms create dimers. Each $\mathrm{Si}$ atom in the dimer is also bonded to two $\mathrm{Si}$ atoms in

* corresponding author; e-mail:

wojciech.koczorowski@put.poznan.pl the atomic layer below. Therefore, the resulting unit cell contains two dangling bonds [5], seen as parallel rows of dimers on the top $\mathrm{Si}(100)$ layer in topographic scanning tunneling microscopy (STM) images [9].

The $\operatorname{Si}(100) 2 \times 1$ substrate is chemically active, with usually larger number of the local defects density, in comparison with the $\mathrm{Si}(111) 7 \times 7$ substrate prepared under the same conditions [5]. The Ni-modified surface exhibits even higher density of the surface defects, but it becomes more chemically stable, in general $[8,10]$. Two kinds of defects, generated by dimer vacancies, had been distinguished on the clean $\mathrm{Si}(100)$ substrate: single and double neighboring dimer defects, referred to as $A$ and $B$ defects, respectively. Both of them show a semiconducting character in local $I-V$ characteristics [5, 7]. Adsorption of dissociated water molecules results in so-called $C$-type defects $[11,12]$. These defects are seen as single or double $\mathrm{Si}$ atom vacancies in neighboring dimers in STM images. The $C$-type defect changes the local electronic properties from semiconducting to the metallic ones, and this fact can be observed by means of the $I-V$ and $\mathrm{d} I / \mathrm{d} V$ spectroscopic curves [5]. The STM/S study of defects of this type on an $\mathrm{Si}(100)$ surface and their evolution into defects of other types at room temperature (RT) was performed by Sobotik and Ostadal [13], and these results quantitatively correspond to the local density of states (LDOS) for $C$ defects calculated by Uda and Terakura [14].

A small amount of $\mathrm{Ni}$ atom contamination, produced e.g. by the contact of the Si substrate with stainless-steel tweezers, can induce different $2 \times n$-type reconstructions of the $\mathrm{Si}(100)$ surface [15]. The evolution of the Ni-contaminated $\mathrm{Si}(100) 2 \times n$ reconstruction due to different parameters of the annealing procedures was studied by Koo et al. in 1995 [8]. The modification of the $\mathrm{Si}(100)$ substrate by deposition of $\mathrm{Ni}$ atoms, followed 
by annealing at the temperature above $800 \mathrm{~K}$, results in the occurrence of parallel lines of defects in the direction perpendicular to the dimer rows [10]. Nickel atoms diffuse into a number of top layers and they are incorporated into the crystal lattice. A small amount of $\mathrm{Ni}$ atoms deposited onto an $\mathrm{Si}(111)$ substrate, followed by the flashing procedure, generates the $\sqrt{19} \times \sqrt{19}$ surface reconstruction, but in this case $\mathrm{Ni}-\mathrm{Si}$ bonds inside the top two layers can be broken by annealing at $970 \mathrm{~K}[16]$.

Although the equilibrium binary Ti-Si phase diagram is very complex [17], in the case of thin layers only $\mathrm{TiSi}_{2}$ has been observed so far on the silicon surface. Two different structural phases, $C 49$ and $C 54$, could be expected to occur as layers of thickness up to $10 \mathrm{~nm} \mathrm{[18].} \mathrm{The}$ former has a base-centered orthorhombic structure, and the latter crystallizes with a face-centered orthorhombic lattice. The temperature of the transition between these phases strongly depends on the layer thickness [18]. Due to its thermal stability and low resistivity $\mathrm{TiSi}_{2}$ is often used for nanoelectronic applications such as electrode or the Schottky diode production. Other forms of $\mathrm{TiSi}_{2}$ were found in the amorphous $\mathrm{Ti} / \mathrm{Si}(100)$ interlayer [19]. The growth of $\mathrm{TiSi}_{2}$ on silicon substrates, especially $\mathrm{Si}(111)$ and $\mathrm{Si}(100)$, had already been studied by STM and scanning tunneling spectroscopy (STS) methods, see e.g. [20, 21]. The density of states for both phases had been calculated theoretically [21, 22]. However, there is no theoretical prediction of LDOS for finite silicide clusters or nanostructures.

Our initial intention was to use the $\operatorname{Si}(100) 2 \times n$ reconstruction generated by $\mathrm{Ni}$ atoms to create an elongated $\mathrm{TiSi}_{2}$ structure on it, and compare the results with the latest measurements on the $\operatorname{Si}(111) \sqrt{19} \times \sqrt{19}$ substrate [16]. We wanted to check the possibility of producing stable nanowires made of the $C 54$ phase grown along and/or between defect lines on the modified Si(100) substrate, and to characterize them by STM method.

\section{Experiment}

Silicon samples prepared from the commercially available wafer were degassed for $12 \mathrm{~h}$ at temperature slightly over $850 \mathrm{~K}$ and flashed five times at a temperature about $1400 \mathrm{~K}$ for $t=10 \mathrm{~s}$ before the $\mathrm{Ni}$ doping procedure. After the last flash the samples were slowly cooled down at the average rate of $1 \mathrm{~K}$ per second. After this preparation procedure the $2 \times 1$ reconstruction was obtained and checked by the STM method. Next, the substrate was doped with a small amount of $\mathrm{Ni}$ atoms (below $0.1 \mathrm{ML}$ ) and flashed at the same parameters. The obtained surfaces were characterized by the STM topography.

Titanium was deposited onto the modified $\mathrm{Si}(100)$ substrate kept at RT in an UHV chamber (about $1 \times$ $10^{-9}$ Torr during the deposition) by means of a commercial Omicron electron beam (EB) evaporator. The Ti coverage was estimated at about $3 \mathrm{ML}$. The STM investigations were performed directly after the deposition, and after the subsequent annealing processes at
$970 \mathrm{~K}$ and $1070 \mathrm{~K}$. The samples were annealed for $30 \mathrm{~min}$ and $20 \mathrm{~min}$, respectively. At the end of each step of the preparation procedure the samples were cooled down intrinsically. The temperature of the samples during the annealing processes was controlled by an optical pyrometer with an accuracy of $10 \mathrm{~K}$. Also, topographic images of samples annealed at $1070 \mathrm{~K}$ were obtained by the atomic force microscopy (AFM) technique with an ultra sharp tip $\left(r_{0}=5 \mathrm{~nm}\right)$ in the contact mode.

All the STM measurements were carried out in UHV conditions at RT. The base pressure in the system was at the level of $2 \times 10^{-10}$ Torr. In all the experiments the composition of the residual gases in the chamber was monitored with a mass spectrometer and it was typical for UHV conditions. The typical measurement parameters were as follows: the bias voltage was stabilized between $\pm 2.5 \mathrm{~V}$, the tunneling current varied from $0.06 \mathrm{nA}$ to $0.5 \mathrm{nA}$, the scanning frequency was kept between $0.5 \mathrm{~Hz}$ and $2 \mathrm{~Hz}$. All the STM measurements were performed with electrochemically etched tungsten tips. The obtained images were processed by the Nanotec WSxM 4.0 software, without filtering [23].

\section{Results}

An STM image of the Si(100) surface with atomically flat terraces is shown in Fig. 1 . The height of the monoatomic terraces was $0.14(4) \mathrm{nm}$ and the spacing between dimers in a row was $0.385(8) \mathrm{nm}$. These values are in good agreement with earlier studies [6, 24].

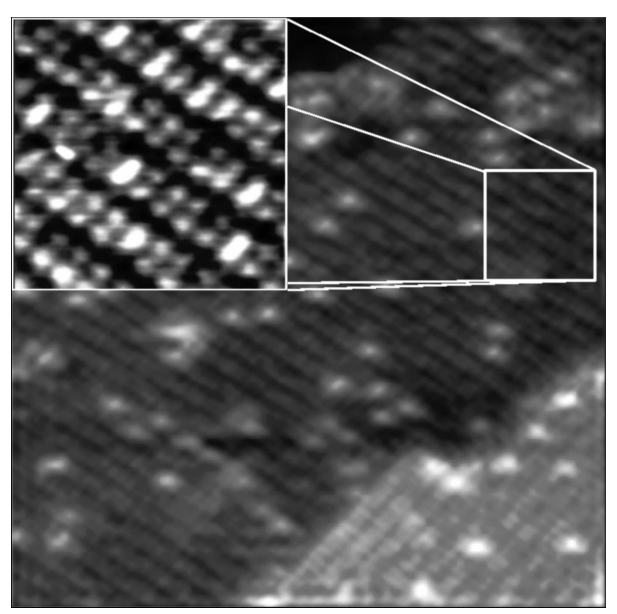

Fig. 1. Atomically flat terraces on $\mathrm{Si}(100)$ substrate $\left(20 \times 20 \mathrm{~nm}^{2}\right)$. Enlarged area $\left(4 \times 4 \mathrm{~nm}^{2}\right)$ shows the $2 \times 1$ dimer reconstruction.

The Ni-modified $\mathrm{Si}(100)$ substrates showed lines of defects similar to those observed by Ono et al. [10]. The defect lines were caused by $\mathrm{Ni}$ atoms which had diffused into top layers of $\mathrm{Si}$ and became bonded there. Each defect line is perpendicular to the dimer rows, and obviously sequentially perpendicular or parallel to the step edges (Fig. 2a). 

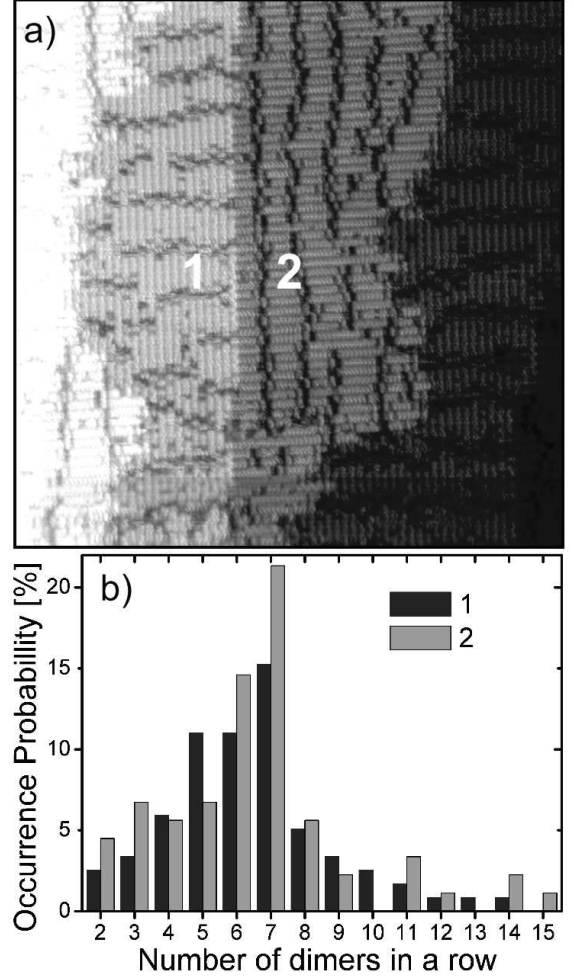

Fig. 2. (a) STM topography image of the Ni-modified $\mathrm{Si}(100)$ substrate $\left(50 \times 50 \mathrm{~nm}^{2}\right)$. (b) Histograms of the non-defected dimer lines obtained for both types of $\mathrm{Si}(100)$ terraces indicated in (a).

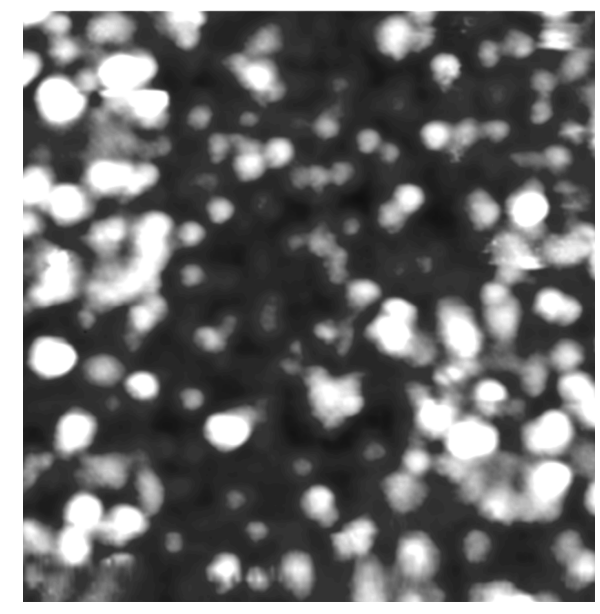

Fig. 3. STM image of titanium layer consisting of Ti clusters deposited onto the $\mathrm{Si}(100)$ substrate $(20 \times$ $\left.20 \mathrm{~nm}^{2}\right)$.

The deposition of titanium on the modified $\mathrm{Si}(100)$ substrate resulted in a full coverage of the substrate, but the top Ti layer was not homogeneous. Rather than a continuous layer, it represented an agglomerate of symmetrical Ti clusters (Fig. 3). The surface root mean square of the roughness was estimated at $0.087 \mathrm{~nm}$. Deposited titanium created clusters of lateral diameter about 1.4(4) nm and with an apparent height up to $0.6 \mathrm{~nm}$ as seen in Fig. 3 .
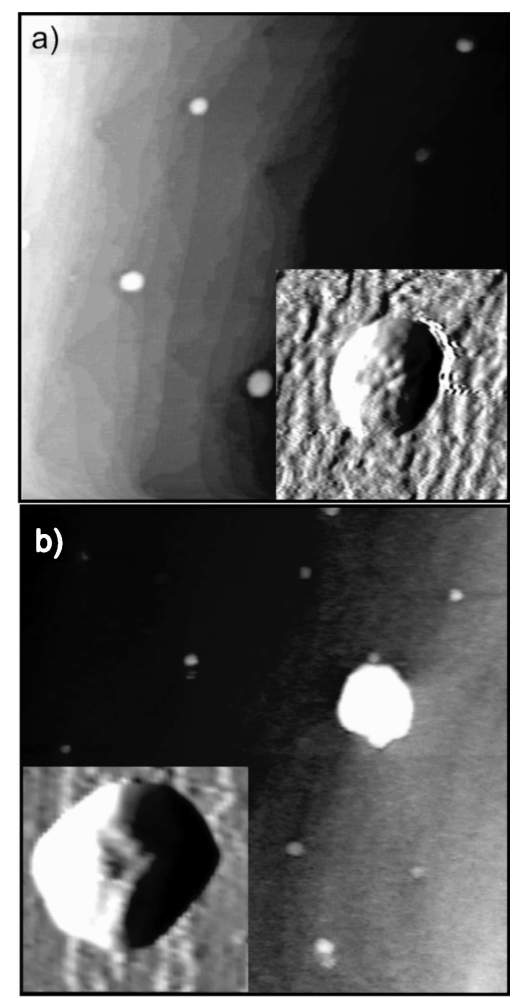

Fig. 4. (a) STM topography image after annealing at $970 \mathrm{~K}\left(400 \times 400 \mathrm{~nm}^{2}\right)$; enlarged topography derivative image of a cluster $\left(40 \times 40 \mathrm{~nm}^{2}\right)$ with visible decoration of the top layer. (b) AFM image $\left(400 \times 400 \mathrm{~nm}^{2}\right)$ after sequence annealing at $1070 \mathrm{~K}$; enlarged STM topography derivative image of a $\mathrm{TiSi}_{2}$ nanostructure $\left(50 \times 50 \mathrm{~nm}^{2}\right)$.

In the next step the samples were annealed at $970 \mathrm{~K}$. Randomly distributed islands of titanium disilicide grown at kinks and step edges appeared. Their average lateral size and height were estimated at about $16 \mathrm{~nm}$ and $1.7 \mathrm{~nm}$, respectively (Fig. 4a). The uncovered original $\mathrm{Si}(100)$ surface was seen between the disilicide islands. A majority of the created $\mathrm{TiSi}_{2}$ nanostructures were decorated on top with Ti clusters, the diameter of which was about 1.50 (4) nm (Fig. 4a).

Annealing at $1070 \mathrm{~K}$ resulted in the formation of two types of structures, visible in the AFM images. The smallest ones had a diameter of about $10 \mathrm{~nm}$ and they were less than $1 \mathrm{~nm}$ in height. Islands of the other type were about three times larger in diameter $(\approx 33(8) \mathrm{nm})$ and their average height was about 5.1(6) nm (Fig. 4b). The uncovered $\mathrm{Si}(100)$ surface, with unaltered lines of defects, was seen in the obtained STM images (inset in Fig. 4b).

\section{Discussion}

Distances among the lines of defects caused by Ni doping varied from 1 to $10 \mathrm{~nm}$. These distances could limit the length and the width of the nanowires or nanostructures of other types created on the modified $\mathrm{Si}(100)$ surface, as the lines of defects can limit their growth. Point defects are also known to act as pinning points for the 
growth of metallic nanowires [25]. Histograms created for terraces of each kind showed that the non-defected dimer rows tend to consist of about 7 dimers each (Fig. 2b). Histograms created for terraces of every kind show that the non-defected dimer rows tend to consist of about 7 dimers each (Fig. 2b). Peaks for the value one, corresponding to single dimers commonly found along the defect lines (about $34 \%$ and $24 \%$ for terraces 1 and 2 , respectively) were omitted in this figure, because one dimer does not create a row. This analysis shows that the reconstruction $2 \times 8$ in this system is the most energetically stable, as it was shown for GeSi layers on the $\mathrm{Si}(100)$ substrate $[26]$.

The samples with the deposited Ti layer were subsequently annealed at $970 \mathrm{~K}$. This temperature was above the threshold for the formation of structural phases $C 49$ and $C 54$ of $\mathrm{TiSi}_{2}$. The phase transition seems to have started [27], as we could observe more symmetrical structures characteristic of the $C 54$ phase. As regards the decoration of the top layer of $\mathrm{TiSi}_{2}$ nanostructures with small clusters, we interpret them as Ti clusters from the top layer (present on the substrate surface before the annealing process) which were in excess of the amount of $\mathrm{Ti}$ necessary to create the stoichiometric $\mathrm{TiSi}_{2}$ structure. The dimensions of these clusters, comparable with those observed after the deposition of $\mathrm{Ti}$, support this explanation. In general, this means that we observed an early stage of the silicide growth.

The final annealing temperature, $1070 \mathrm{~K}$, was above the threshold at which the transition to the $C 54$ phase should be completed. The AFM images showed two kinds of nanostructures (Fig. 4). The type I nanostructures were slightly smaller than the structures obtained in the previous annealing process. This could suggest a partial dissolution of the previous nanostructures into the substrate. On the other hand, the surface had not changed and still presented the same $2 \times n$ reconstruction as induced before Ti deposition. This observation led us to the conclusion that $\mathrm{Ni}$ atoms in the system under investigation were strongly bonded within the top layers of $\mathrm{Si}(100)$ in comparison with the $\operatorname{Si}(111) \sqrt{19} \times \sqrt{19}$ substrate. In this latter case alloying of $\mathrm{Si}, \mathrm{Ti}$ and $\mathrm{Ni}$ atoms was observed after annealing at similar temperatures [16]. This is a strong indication that the nanostructures formed in our experiment were composed of $\mathrm{TiSi}_{2}$. At the same time, type II nanostructures, much larger than type I nanostructures, had appeared, most probably as a result of the merging of smaller ones due to enhanced surface diffusion. This type of dynamic coalescence is well known [28]. Considering the preparation conditions, we can say that embryo structures of the $C 54$ phase were created and observed in this case. Presence of two types of nanostructures could be explained in this way that the transformation between two phases was not accomplished in this case and we observed still structures made of $C 49$ (smaller ones) and $C 54$ (larger ones) phases. Unfortunately, no elongated form, and in particular no nanowires, could be found on the surface.

\section{Conclusions}

The deposition of a small amount of Ni onto the $\mathrm{Si}(100)$ substrate, followed by the flashing procedure, resulted in the formation of lines of missing Si dimers at the $2 \times 1$ reconstructed $\mathrm{Si}(100)$ surface. Nanostructures of titanium silicides were grown by deposition of Ti onto the modified $\mathrm{Si}(100)$ substrate followed by the post-deposition annealing at temperatures ranging from $970 \mathrm{~K}$ to $1070 \mathrm{~K}$.

The creation of titanium disilicide nanoislands via the above-mentioned annealing did not affect the structure of the modified substrate, which leads to the conclusion that the nanostructures were composed only of $\mathrm{TiSi}_{2}$ (without Ni impurities) in phases $C 49$ and $C 54$. The number of nanostructures of the $C 54$ phase was found to increase with the annealing temperature.

However, the linear defects created on the $\mathrm{Si}(100)$ $2 \times 1$ substrate failed to induce the growth of $C 54$ phase nanowires.

\section{Acknowledgments}

This study was supported by the Polish Ministry of Science and Higher Education under the project No. N-N202-169236.

\section{References}

[1] A. Wawro, S. Suto, R. Czajka, A. Kasuya, Nanotechnology 19, 205706 (2008).

[2] A. Wawro, S. Suto, A. Kasuya, Phys. Rev. B 72, 205302 (2005).

[3] P. Avouris, I.-W. Lyo, Science 264, 942 (1994).

[4] M.W. Radny, P.V. Smith, L. Jurczyszyn, Phys. Rev. B 81, 085424 (2010).

[5] R. Wiesendanger, Scanning Probe Microscopy and Spectrometry: Methods and Applications, Cambridge University Press, Cambridge 1994.

[6] R.J. Hamers, R.M. Tromp, J.E. Demuth, Phys. Rev. B 34, 5343 (1985).

[7] R.J. Hamers, Ph. Avouris, F. Bozso, Phys. Rev. Lett. 59, 2071 (1987).

[8] J.-Y. Koo, J.-Y. Yi, Ch. Hwang, D.-H. Kim, S. Lee, Phys. Rev. B 52, 17269 (1995).

[9] R.M. Tromp, R.J. Hamers, J.E. Demuth, Phys. Rev. Lett. 55, 1303 (1985).

[10] I. Ono, M. Yoshimura, K. Ueda, J. Vac. Sci. Technol. B 16, 2947 (1998).

[11] M.Z. Hossain, Y. Yamashita, K. Mukai, J. Yoshinobu, Phys. Rev. B 67, 153307 (2003).

[12] S. Okano, A. Oshiyama, Surf. Sci. 554, 272 (2004).

[13] P. Sobotik, I. Ostadal, Surf. Sci. 602, 2835 (2008).

[14] T. Uda, K. Terakura, Phys. Rev. B 53, 6999 (1996).

[15] K. Kato, T. Ide, S. Miura, A. Tamura, T. Ichinokawa, Surf. Sci. 194, L87 (1988).

[16] M. Cegiel, M. Bazarnik, P. Biskupski, S. Winiarz, J. Gutek, A. Bos, S. Suto, S. Mielcarek, A. Wawro, R. Czajka, Appl. Surf. Sci. 254, 6948 (2008). 
[17] Silicide Technology for Integrated Circuits, Eds. Z. Ma, L.H. Allen, L.J. Chen, The Institution of Electrical Engineers, London 2004.

[18] H. Jeon, G. Yoon, R.J. Nemanich, Thin Solid Films 299, 178 (1997).

[19] T.H. Yang, L.J. Chen, J. Appl. Phys. 98, 034302 (2005).

[20] I. Goldfarb, S. Grossman, G. Cohen-Taguri, M. Levinshtein, Appl. Surf. Sci. 238, 29 (2004).

[21] M. Toramaru, T. Iida, K. Sato, S. Ohno, K. Shudo, Y. Morikawa, M. Tanaka, J. Phys., Condens. Matter 20, 485006 (2008).

[22] L.F Mattheiss, J.C. Hensel, Phys. Rev. B 39, 7754 (1989).
[23] I. Horcas, R. Fernández, J.M. Gómez-Rodríguez, J. Colchero, J. Gómez-Herrero, A.M. Baro, Rev. Sci. Instrum. 78, 013705 (2007).

[24] G.D. Wilk, Y. Wei, H. Edwards, R.M. Wallance, Appl. Phys. Lett. 70, 2388 (1997).

[25] P. Kocan, L. Jurczyszyn, P. Sobotik, I. Ošt'ádal, Phys. Rev. B 77, 113301 (2008).

[26] R. Butz, S. Kampers, Appl. Phys. Lett. 61 (1992).

[27] Z. He, M. Stevens, D.J. Smith, P.A. Bennett, Surf. Sci. 524, 148 (2003).

[28] M. Zinke-Allmang, L.C. Feldman, M.H. Grabow, Surf. Sci. Rep. 16, 377 (1992). 